\title{
Zwischen Führungsrolle und Sprachlosigkeit: Europas Lehren aus dem Klimagipfel in Kopenhagen
}

\author{
Severin Fischer und Jo Leinen*
}

Totales Scheitern oder kleiner Erfolg? Fortschritt oder Rückschritt? Absehbarer Minimalkompromiss oder überraschend positives Resultat? Die zutreffende Bewertung des Ergebnisses der Kopenhagener Klimakonferenz (COP 15) ${ }^{1}$ wird noch lange Zeit umstritten bleiben und wesentlich vom weiteren Verlauf der Verhandlungen in den Jahren 2010 und 2011 abhängen. In jedem Fall wird der Klimagipfel vom 7. bis 19. Dezember 2009 als bislang größte internationale Konferenz des 21. Jahrhunderts in die Geschichtsbücher eingehen. Nie zuvor haben Klimaschutzfragen ein so breites öffentliches Interesse hervorgerufen und nie zuvor war es möglich gewesen, rund 120 Staats- und Regierungschefs zu einer gemeinsamen Debatte über einen globalen Umweltschutzvertrag zu bewegen. Obgleich mit der Kopenhagener Konferenz ein neuer Superlativ medialer Aufmerksamkeit erreicht wurde, schienen die Delegationen der 192 Parteien der Klimarahmenkonvention UNFCCC ${ }^{2}$ nicht in der Lage oder nicht gewillt zu sein, die zwölftägige Konferenz mit einem völkerrechtlich bindenden Vertrag abzuschließen. Am Ende einer nicht enden wollenden Verhandlungsnacht nahm das Plenum der Konferenz am 19. Dezember 2009 ein kurzes und wenig ehrgeiziges Dokument nicht formell an, sondern lediglich unverbindlich ,zur Kenntnis ‘. 3 Damit wurde zwar der Prozess im Rahmen der UN-Klimaverhandlungen am Leben erhalten, vom erklärten Ziel, sich auf ein völkerrechtlich verbindliches Abkommen zu einigen, blieb die Konferenz jedoch meilenweit entfernt.

Das Scheitern der Klimaverhandlungen war nicht nur enttäuschend für die zahlreichen Umweltaktivisten und Klimawissenschaftler, die über viele Jahre hinweg auf die Bedeutung und Dringlichkeit eines Abschlusses der internationalen Verhandlungen hingewiesen hatten. Es war auch ein unbefriedigendes Ergebnis für die Europäische Union, die sich mit ihrem ambitionierten Klimaschutzprogramm und ihrer Verhandlungsstrategie nicht durchsetzen konnte. Eine erste Analyse mag zu dem Schluss verleiten, dass es Europa an der notwendigen Zahl internationaler Partner mangelte, die es für eine Einigung hätte gewinnen müssen. Doch kann dieser Ansatz alleine als Erklärung für den wenig erfolgreichen Verlauf der Konferenz aus Sicht der Europäischen Union ausreichen?

Es erscheint angebracht, einen Blick auf die Rolle der Europäischen Union in den internationalen Klimaverhandlungen zu werfen, deren praktische Ausgestaltung zu analysieren und daraus Lehren für den fortlaufenden Post-Kopenhagen-Prozess zu ziehen. Gleichzeitig stellen sich nach dem wenig hoffnungsvollen Ende der Gespräche noch weitere grundsätzli-

\footnotetext{
Severin Fischer, Wissenschaftlicher Mitarbeiter, Institut für Europäische Politik, Berlin. Forscht im Rahmen eines gemeinsamen Projekts mit der ASKO EUROPA-STIFTUNG zu Fragen europäischer Energie- und Klimapolitik.

Jo Leinen, MdEP, Vorsitzender des Ausschusses für Umweltfragen, Volksgesundheit und Lebensmittelsicherheit im Europäischen Parlament und Leiter der Delegation des Europäischen Parlaments bei der Klimakonferenz in Kopenhagen.

1 „15. Vertragsstaatenkonferenz der Klimarahmenkonvention und 5. Vertragsstaatenkonferenz des Kyoto-Protokolls (COP 15/CMP 5)“.

2 „United Nations Framework Convention on Climate Change“.

3 UNFCCC: Copenhagen Accord. Decision -/CP.15, advance unedited version, 19.12.2009, abrufbar unter: http://unfccc.int/files/meetings/cop_15/application/pdf/cop15_cph_auv.pdf (letzter Zugriff: 15.02.2010).
} 
chere Fragen, etwa zur künftigen Rolle der Vereinten Nationen sowie den Grenzen internationaler Problemlösungsfähigkeit.

\section{Internationale Klimapolitik von Rio bis Kopenhagen}

Seit dem Abschluss der UN-Konferenz in Rio de Janeiro 1992 und der damit verbundenen Gründung der Klimarahmenkonvention, steht der Klimawandel auf der Agenda der Vereinten Nationen. Die Kopenhagener Klimakonferenz stellte in diesem Kontext das 15. Treffen der Vertragsparteien (COP 15) seit Inkrafttreten der UN-Konvention im Jahr 1994 dar. ${ }^{4}$ Im Kontext der UNFCCC wurde auf dem 3. Treffen der Vertragsparteien im Jahr 1997 der erste internationale Klimaschutzvertrag geschlossen - das Kyoto-Protokoll. ${ }^{5}$ Allerdings konnte erst durch die Ratifizierung in der russischen Duma im Jahr 2004 die notwendige Hürde für sein Inkrafttreten überschritten werden. ${ }^{6}$ Die Vereinigten Staaten lehnten bis zuletzt eine Ratifikation des Klimavertrags ab, der erstmals absolute Reduktionsziele für Industrieländer im Zeitraum 2008 bis 2012 vorsah. Durch das Ende der ersten Verpflichtungsperiode des Kyoto-Protokolls im Jahr 2012 und durch die Vielzahl neuer wissenschaftlicher Erkenntnisse des ,Weltklimarates' IPCC, ${ }^{7}$ der in seinem 4. Sachstandsbericht aus dem Jahr 2007 einen noch dramatischeren Klimawandel prognostiziert hatte, war die Dringlichkeit für eine Ausweitung und Verbesserung des internationalen Klimaregimes weiter erhöht worden. ${ }^{8}$

Auf dem Klimagipfel 2007 war von den Vertragsstaatenparteien der Klimarahmenkonvention die ,Bali Road Map“ und die damit verbundene Einsetzung von zwei Arbeitsgruppen beschlossen worden, deren Aufgabe es fortan sein sollte, bis zum Gipfel in Kopenhagen einen neuen völkerrechtlichen Rahmen auszuarbeiten, der den wissenschaftlichen Vorgaben des IPCC entsprechen sollte. ${ }^{9}$ Ausgestattet mit diesem Mandat begannen die Verhandlungen für COP 15. Nach einem ergebnislosen Zwischenstopp in Poznań Ende 2008, sollte in Kopenhagen der wichtige Schritt zur Schaffung eines internationalen Rahmens für den Klimaschutz gefunden werden.

Die Europäische Union war im Kontext der Entwicklung internationaler Übereinkünfte zum Klimaschutz ein vergleichsweise aktiver und ehrgeiziger Akteur. Im Gegensatz zu anderen Industriestaaten verpflichtete sich die Gemeinschaft der EU-15 im Rahmen des Kyoto-Protokolls zu einem kollektiven Reduktionsziel von 8 Prozent in der ersten Verpflichtungsperiode. Dieses gemeinsame Auftreten der Europäischen Union bei den Klimaverhandlungen setzte sich auch in den kommenden Jahren fort. Zwar sind bis heute

4 Das „Rahmenübereinkommen der Vereinten Nationen über Klimaänderungen“ (UNFCCC) wurde am 9. Mai 1992 in New York unterzeichnet und trat nach einem Ratifikationsprozess am 21. März 1994 in Kraft. Der Text der Klimarahmenkonvention ist verfügbar unter: http://unfccc.int/resource/docs/convkp/convger.pdf (letzter Zugriff: 15.02.2010).

5 Der Text des Kyoto-Protokolls (,Kyoto Protocol to the United Nations Framework Convention on Climate Change") ist verfügbar unter: http://unfccc.int/resource/docs/convkp/kpeng.pdf (letzter Zugriff: 15.02.2010).

6 Für das Inkrafttreten des Kyoto-Protokolls musste eine doppelte Hürde überschritten werden: Es bedurfte der Ratifikation durch 55 Staaten, die 55 Prozent der Emissionen aus Annex I-Staaten umfassen (Art. 25 Abs. 1 Kyoto-Protokoll). Als Annex I-Staaten werden jene Staaten bezeichnet, die in Anlage I zur Klimarahmenkonvention aufgeführt sind und sich durch die Klimarahmenkonvention zur Einhaltung quantifizierbarer Emissionsobergrenzen verpflichtet haben.

7 „Intergovernmental Panel on Climate Change“.

8 Vgl. Intergovernmental Panel on Climate Change: Climate Change 2007: Synthesis Report. Contribution of Working Groups I, II and III to the Fourth Assessment Report of the Intergovernmental Panel on Climate Change, Genf 2007.

9 Vgl. UNFCCC: Bali Action Plan. Decision -/CP.13, advance unedited version, Bali 2007, abrufbar unter: http://unfccc.int/files/meetings/cop_13/application/pdf/cp_bali_action.pdf (letzter Zugriff: 08.02.2010). 
die Mitgliedstaaten Vertragsparteien der Klimarahmenkonvention und des Kyoto-Protokolls, doch gilt gemeinhin, dass die Europäische Union bei den Verhandlungen gemeinschaftlich von der rotierenden Ratspräsidentschaft und der Europäischen Kommission vertreten wird. ${ }^{10}$

Die Gemeinschaftsmethode in der Europäischen Union liegt zum einen in der vertraglich geregelten Kompetenzordnung im Bereich der Umweltpolitik begründet. Zum anderen hat das kollektive Auftreten seine Ursache in der internen Politikfeldgestaltung der EU-Klimapolitik. Nach dem Wortlaut der Verträge teilen sich die Gemeinschaft und die Mitgliedstaaten die Zuständigkeit in der Klimapolitik. ${ }^{11}$ Mit der Entscheidung zur Einsetzung eines europaweiten Emissionshandelssystems zur Steuerung des $\mathrm{CO}_{2}$-Ausstosses wurde die Einflussmöglichkeit der Mitgliedstaaten zudem weiter eingeschränkt. ${ }^{12}$ Der gemeinschaftliche Ansatz wurde auch im Vorfeld der Kopenhagener Klimakonferenz gewahrt, sodass die schwedische Ratspräsidentschaft die Verhandlungsführung im Namen der Europäischen Union übernahm.

Als einziger Akteur der internationalen Klimaverhandlungen war die Europäische Union mit einem rechtsverbindlichen Klimaschutzkonzept nach Kopenhagen gereist. Sowohl intern, durch die Verabschiedung des Energie- und Klimapakets im Dezember 2008, als auch extern, durch die Formulierung eines Verhandlungsmandats von Seiten des Europäischen Rates, war die Europäische Union mit konkreten Vorschlägen in die Gespräche gegangen. Die strategische Führungsrolle bei den Verhandlungen sollte dementsprechend in erster Linie durch ehrgeizige Klimaschutzvorgaben in Europa wahrgenommen und in einem zweiten Schritt durch Finanzierungszusagen für den internationalen Klimaschutz untermauert werden. Hinzu kam das Angebot, die eigenen klimaschädlichen Treibhausgasemissionen um 30 Prozent gegenüber 1990 zu reduzieren, sollten andere große Industrie- und Schwellenländer ebenfalls Reduktionszielen zustimmen.

\section{Auf dem Weg nach Kopenhagen: die Verhandlungen im Vorfeld zu COP 15}

Durch das Verhandlungsmandat des Klimagipfels in Bali im Jahr 2007 war die Dramaturgie für die folgenden zwei Jahre weitgehend festgelegt worden. Die technischen Lösungsansätze der zwei Arbeitsgruppen, der „Ad Hoc Working Group on Long-term Cooperative Action under the Convention“ (AWG-LCA) und der „Ad Hoc Working Group on Further Commitments for Annex I Parties under the Kyoto Protocol“" (AWG-KP), sollten bis zur Kopenhagener Konferenz konkrete Vorschläge zu den zahlreichen Detailfragen sammeln und erste Lösungsansätze entwickeln. Dies würde die Arbeit in den zwei Konferenzwochen in Kopenhagen erleichtern und die Möglichkeit bieten, die Beschlüsse umgehend in ein völ-

10 Vgl. Alexandra Lindenthal: Leadership im Klimaschutz. Die Rolle der Europäischen Union in der internationalen Klimapolitik, Frankfurt/New York 2009.

11 Während Klimapolitik im Vertrag von Nizza noch nicht explizit benannt wurde und die internationale Kooperation zwischen der Gemeinschaft und den Mitgliedstaaten unter Art. 174 Abs. 4 Vertrag zur Gründung der Europäischen Gemeinschaft (EGV) subsumiert wurde, wird im Vertrag von Lissabon eine geteilte Zuständigkeit zwischen Union und Mitgliedstaaten festgehalten (Art. 4 Abs. 2 Lit. e Vertrag über die Arbeitsweise der Europäischen Union, AEUV), das Problem des Klimawandels erstmals explizit benannt (Art. 191 Abs. 1 AEUV) und die geteilte Zuständigkeit in internationalen Verhandlungen bestätigt (Art. 191 Abs. 4 AEUV). Vgl. auch Severin Fischer: Energie- und Klimapolitik im Vertrag von Lissabon: Legitimationserweiterung für wachsende Herausforderungen, in: integration 1/2009, S. 50-62.

12 Das EU-Emissionshandelssystem trat am 1. Januar 2005 in Kraft. Mit Beginn der dritten Handelsperiode am 1. Januar 2013 wird das System ohne die Verwendung Nationaler Allokationspläne gesteuert. Vgl. Richtlinie 2009/29/EG des Europäischen Parlaments und des Rates vom 23. April 2009 zur Änderung der Richtlinie 2003/87/EG zwecks Verbesserung und Ausweitung des Gemeinschaftssystems für den Handel mit Treibhausgasemissionszertifikaten, in: Amtsblatt der EU, Nr. L 140 vom 5. Juni 2009, S. 63-87. 
kerrechtlich verbindliches Format zu transformieren. Die Arbeitsgruppen nahmen nach der Konferenz in Bali ihre Arbeit auf und entwickelten erste Strukturen für einen Kopenhagener Klimavertrag sowie eine zweite Verpflichtungsperiode für die Annex-I-Staaten im Rahmen des Kyoto-Protokolls. Doch bereits die Trennung der Arbeitsgruppen und die damit verbundene Aufteilung der Welt in zwei unterschiedlich stark verantwortliche Staatengruppen, sorgte für Unmut unter den Verhandlungspartnern. ${ }^{13}$

Während die Verhandlungen auf technischer Ebene voranschritten, hatten die Regierungen ausreichend Zeit ihre internationalen Verpflichtungen im nationalen Kontext vorzubereiten. Dies galt insbesondere für diejenigen Staaten, die das Kyoto-Protokoll nicht oder zu diesem Zeitpunkt noch nicht ratifiziert hatten, jedoch Bereitschaft signalisierten, sich an einem zukünftigen Klimaabkommen beteiligen zu wollen (USA, Australien). Ebenso sollten diejenigen Staaten, die ihre Zusagen im Rahmen der ersten Verpflichtungsperiode nicht erfüllt hatten oder absehbar nicht erfüllen würden, Maßnahmen einleiten, um robust und glaubwürdig in die Verhandlungen zu gehen (Japan, Kanada). Schließlich wurden auch erstmals Schwellenländer aufgefordert, Angebote zu unterbreiten, wie sie ihre stetig steigenden Emissionen begrenzen und ihre Volkswirtschaften klimaverträglicher gestalten wollen. Spätestens seit den Empfehlungen des IPCC im Rahmen seines 4. Sachstandsberichts im Jahr 2007 zeichnete sich ab, dass das Erreichen des $2{ }^{\circ} \mathrm{C}$-Ziels ohne die Beteiligung der größten Schwellenländer unwahrscheinlich sein würde.

Trotz des strukturierten Fahrplans durch die ,Bali Road Map“ und der absehbaren Notwendigkeit, mit nationalen Angeboten nach Kopenhagen reisen zu müssen, um einen Kompromiss erreichen zu können, bedurfte es einer Initialzündung auf internationaler Ebene, damit der Zug nach Kopenhagen ins Rollen kommen würde. Nach monatelangen Blockaden und wenig substanziellen Fortschritten kann rückblickend die Wahl des US-amerikanischen Präsidenten Barack Obama im November 2008 als ein wichtiger Meilenstein der Verhandlungen bezeichnet werden. Durch den Wiedereinstieg der USA in die internationale Klimapolitik und die Ankündigung des US-Präsidenten, den Klimawandel (neben der nationalen Gesundheitsreform) zum Schwerpunkt seiner Amtszeit zu machen, war auch für andere Akteure Bewegung aus der bis dahin festgefahrenen Position heraus möglich. Auch in anderen wichtigen Industriestaaten sorgten Wahlerfolge von Oppositionsparteien für Hoffnung in der Klimapolitik. So gelang es unter anderem Kandidaten in Australien oder Japan einen Regierungswechsel herbeizuführen, indem sie den Kampf gegen den Klimawandel und eine Rückkehr auf die internationale Bühne der Klimapolitik versprachen.

Von Seiten der Industriestaaten war durch die mehrheitliche Bereitschaft zum Klimaschutz eine erste Vorleistung für die Kopenhagener Verhandlungen erbracht worden. Dennoch zögerten viele Entwicklungs- und Schwellenländer aufgrund der Unsicherheit über die legislative Implementierung der Vorgaben und die finanzielle Ausstattung der internationalen Klimaschutzmaßnahmen, eigene Angebote zu unterbreiten. In der Tat ließ eine Klimaschutzinitiative im US-Kongress lange auf sich warten. Erst im Laufe des Jahres 2009 begannen die Verhandlungen über ein Klimagesetz in den beiden Häusern des Kongresses, sodass bereits zu diesem Zeitpunkt ein Abschluss der Gesetzgebung bis zum Klimagipfel in Kopenhagen unwahrscheinlich erschien. Auch in anderen Staaten wie Australien, Japan, Kanada oder Neuseeland verpassten die Regierungen die Chance, eine rechtsverbindliche Klimaschutzgesetzgebung zu verabschieden, um damit ihre Glaubwürdigkeit international

13 So hatten die USA bereits früh erklärt, sie würden auch einer zweiten Verpflichtungsperiode im Rahmen des Kyoto-Protokolls nicht beitreten. Die Europäische Union blieb bei dieser Frage hingegen lange Zeit unentschieden. 
zu dokumentieren. Bis zum Beginn der Verhandlungen in Kopenhagen blieb die Europäische Union der einzige große Vertreter der Industriestaaten, der mit einem rechtsverbindlichen Klimaschutzprogramm in die dänische Hauptstadt reiste.

Die Unverbindlichkeit der Zusagen von Seiten der Industriestaaten und die mangelnde Bereitschaft der Schwellenländer, sich verbindlichen Klimaschutzzielen zu verpflichten, leitete eine neue Spirale der gegenseitigen Blockaden und Vorwürfe ein, die sich über das Jahr 2009 durch die internationale Klimapolitik zog. Seinen vorläufigen Höhepunkt fand dieses gegenseitige Belauern auf dem Wirtschaftsgipfel der asiatisch-pazifischen Staaten (APEC) im November 2009 in Singapur. Beinahe einmütig bekannten sich die Regierungschefs aus Asien und den USA zu einer Verschiebung der Entscheidung über ein rechtsverbindliches Abkommen auf einen späteren Zeitpunkt nach Kopenhagen.

Auch wenn der Kopenhagen-Prozess nach dem APEC-Gipfel von vielen Beobachtern als gescheitert bezeichnet wurde, zeigten sich einige Staaten von den Entwicklungen dennoch unbeeindruckt. So kündigte Brasilien kurz nach Abschluss der Gespräche in Singapur an, seine Emissionen gegenüber einem Business-as-usual-Szenario um 33 bis 39 Prozent reduzieren zu wollen. Dem brasilianischen Vorschlag folgten Südkorea und weitere Schwellenländer mit der konkreten Benennung ihrer Maßnahmenpakete. Diese Schritte stellten einen Bruch mit der bislang gültigen Sprachregelung dar, wonach sich nur Industriestaaten absoluten Reduktionszielen zu verpflichten hätten. Damit wuchs auch der Druck auf die großen Schwellenländer China und Indien. Schließlich kamen sowohl aus Peking wie aus NeuDelhi wenige Tage vor Beginn der Verhandlungen in Kopenhagen erstmals konkrete nationale Klimaschutzvorschläge. Beide Staaten wollten künftig die $\mathrm{CO}_{2}$-Intensität ihrer Wirtschaft in relativen Größen verringern. Damit hatten sie zwar erstmals ein Ziel formuliert, durch seine Orientierung am Wirtschaftswachstum und die damit verbundene Unwägbarkeit blieben bei vielen Delegationen auf dem Weg nach Kopenhagen ernsthafte Zweifel, ob mit diesen Zielsetzungen die Vergleichbarkeit zu Maßnahmen anderer Schwellenländer oder der Industrieländer aufrechterhalten werden könnte.

\section{Das Verhandlungsmandat der Europäischen Union für Kopenhagen: interne Kon- fliktlösungsansätze als Präzedenzfälle für die Klimakonferenz?}

Während über das Konfliktpotenzial der internationalen Verhandlungen schon zu einem frühen Zeitpunkt berichtet wurde, gingen Interessengegensätze innerhalb der Europäischen Union in der öffentlichen Debatte weitgehend unter. Da durch die Beschlüsse zum Energieund Klimapaket vom Dezember 2008 erste Verhandlungsleitlinien vorgezeichnet waren (so etwa das konditionierte 20- beziehungsweise 30-Prozent-Ziel der Europäischen Union), war es vor allem die Finanzierung von Klimaschutz- und Anpassungsmaßnahmen in Entwicklungs- und Schwellenländern, die innerhalb der Europäischen Union für teils heftige Diskussionen sorgten.

Zur Einleitung eines EU-internen Meinungsbildungsprozesses veröffentlichte die Europäische Kommission im Januar 2009 eine Mitteilung unter dem Titel „Ein umfassendes Klimaschutzübereinkommen als Ziel für Kopenhagen“, die sich vor allem auf die drei Schwerpunkte der internationalen Verhandlungen konzentrierte: Reduzierungsmaßnahmen und -angebote, Klimafinanzierung und Aufbau eines globalen Kohlenstoffmarktes. ${ }^{14} \mathrm{Im}$ September 2009 wurde die Kommissionsvorlage durch ein weiteres Dokument zur interna-

14 Vgl. Europäische Kommission: Mitteilung der Kommission an das Europäische Parlament, den Rat, den Europäischen Wirtschafts- und Sozialausschuss und den Ausschuss der Regionen. Ein umfassendes Klimaschutzübereinkommen als Ziel für Kopenhagen, KOM (2009) 39. 
tionalen Klimafinanzierung ergänzt, das erstmals von einem internationalen Finanzierungsbedarf in Höhe von rund 100 Milliarden Euro jährlich ab 2020 spricht. ${ }^{15}$ Die Kommission formulierte in beiden Papieren die Ansicht, dass die Voraussetzung für das erfolgreiche Fortsetzen der Maßnahmen in der Europäischen Union ein rechtsverbindliches Abkommen als Ergebnis der Verhandlungen in Kopenhagen sei.

Der Umweltministerrat hatte sich zum Ziel gesetzt, bereits im Oktober ein vollständiges Verhandlungsmandat der Europäischen Union für die Kopenhagener Verhandlungen vorzulegen. Auch wenn in den meisten Punkten früh Einigkeit erzielt werden konnte, so etwa zu den Erwartungen an mittel- und langfristige globale Emissionsreduktionen, zur Umsetzung von Anpassungsmaßnahmen oder zu den nächsten Schritten hin zur Entwicklung eines globalen Kohlenstoffmarktes, blieben einige Fragen auch auf dem abschließenden Umweltministerrat am 21. Oktober 2009 ungeklärt. Zu den konfliktträchtigsten Themen zählten insbesondere Europas Beitrag zur internationalen Klimafinanzierung sowie die EU-interne Lastenteilung. Ungeklärt und umstritten blieb jedoch auch die EU-Position zu Emissionsreduzierungen im internationalen Luft- und Seeverkehr, zur Art und Weise der Einbeziehung von Wäldern in ein globales Klimaschutzsystem und zu den Erwartungen an die rechtliche Ausgestaltung eines künftigen Abkommens. Ein weiteres Konfliktfeld zeichnete sich beim Umgang mit Emissionszertifikaten aus dem Kyoto-Protokoll, den sogenannten ,Assigned Amount Units' (AAUs), ab. Infolge der Deindustrialisierung in Mittel- und Osteuropa nach 1990 hatten die zehn neuen EU-Mitglieder teils drastische strukturell bedingte Emissionsreduktionen zu verzeichnen. Gleiches galt im Übrigen für Russland und die Ukraine, deren absolute Gesamtmenge an Reduktionen diejenige der neuen EU-Mitgliedstaaten noch überstieg. Trotz weitreichender Minderungsziele im Rahmen des Kyoto-Protokolls blieben diese Transformationsstaaten gegenüber dem Basisjahr 1990 unterhalb der geforderten Grenze und hatten somit das Recht, überschüssige Zertifikate an diejenigen Staaten zu veräußern, die ihre Ziele nicht erfüllt hatten. Polen und einige andere mittel- und osteuropäische Staaten wehrten sich vehement gegen einen Verzicht auf den Verkauf der Zertifikate, wie er von den anderen Mitgliedstaaten gefordert wurde. Auch diese Entscheidung mit weitreichender Signalwirkung auf die internationalen Verhandlungen musste auf das Gipfeltreffen der Staatsund Regierungschefs, den Europäischen Rat vom 29./30. Oktober 2009, vertagt werden.

Der Europäische Rat hatte sich auf seinem Oktobergipfel zahlreichen, teilweise äußerst komplexen Fragestellungen zu den Leitlinien für das Verhandlungsmandat in Kopenhagen zu widmen. Es gelang den Staats- und Regierungschefs schließlich nicht, sich bei der vielleicht wichtigsten Frage, der Ausgestaltung und Höhe einer EU-Klimafinanzierung, auf einen Kompromiss zu einigen. Zwar wurde dem Gesamtkonzept des Umweltministerrats für das Verhandlungsmandat zugestimmt, die entscheidende Frage der Klimafinanzierung ließ sich jedoch nicht lösen. Die mittel- und osteuropäischen Staaten sowie Italien weigerten sich, im Kontext der internationalen Finanz- und Wirtschaftskrise zusätzliche Mittel bereitzustellen. Deutschland blieb lange unentschieden bis ablehnend. Schließlich bezifferte der Europäische Rat zwar den globalen Finanzierungsbedarf für die Jahre 2010 bis 2012 auf jährlich 5 bis 7 Milliarden Euro und bestätigte die Berechnungen der Kommission, wonach ab 2020 rund 100 Milliarden Euro pro Jahr für die Finanzierung von Klimaschutz- und Anpassungsmaßnahmen bereitgestellt werden müssten. Bei der Nennung eines europäischen Beitrags zu dieser Summe kamen die Staats- und Regierungschefs jedoch nicht über die For-

15 Vgl. Europäische Kommission: Mitteilung der Kommission an das Europäische Parlament, den Rat, den Europäischen Wirtschafts- und Sozialausschuss und den Ausschuss der Regionen. Mehr internationale Finanzmittel für den Klimaschutz: europäisches Konzept für die Kopenhagener Vereinbarung, KOM (2009) 475. 
mulierung eines ,angemessenen Beitrag[s]“ hinaus. Auch über die EU-interne Lastenteilung sowie die Frage des künftigen Umgangs mit den AAUs konnte keine Einigung erzielt werden. ${ }^{16}$

Während sich der Europäische Rat noch um eine einheitliche Position bemühte, formulierte eine andere Institution ihre Erwartungen an den Kopenhagener Gipfel weitaus klarer. Das Europäische Parlament verabschiedete am 25. November 2009 eine Resolution, die mit der Erwartung eines europäischen Beitrags zur Klimafinanzierung in Höhe von 30 Milliarden Euro jährlich erstmals explizite Summen nannte. ${ }^{17}$ Konkretere Zielvorgaben wurden auch für die Emissionsreduktionen aus dem internationalen Luft- und Seeverkehr genannt. Insgesamt zeigte das Parlament mehr Bereitschaft, für einen Kompromiss in Kopenhagen auch neue und innovative Wege zu beschreiten und setzte damit insbesondere den Rat unter Druck, seine Angebote nachzubessern. Die Parlamentarier erlangten zudem infolge eines anderen Ereignisses eine bessere Position in den Gesprächen mit Rat und Kommission: Durch das Inkrafttreten des Lissabon-Vertrags am 1. Dezember 2009 erhielt das Parlament zum einen mehr Informationsrechte vor und während internationalen Verhandlungen. Gleichzeitig ist die Zustimmung der Parlamentarier zu einem internationalen Vertrag im Bereich Umwelt - so etwa einem möglichen Klimaabkommen - von nun an notwendig. ${ }^{18}$

Nach dem Oktobergipfel der Staats- und Regierungschefs verblieb den europäischen Institutionen noch eine letzte Gelegenheit, der Weltklimakonferenz einen weiteren Schub zu verleihen. Am 10./11. Dezember 2009, bereits einige Tage nach Beginn der Verhandlungen in Kopenhagen, fand in Brüssel ein weiteres Treffen der Staats- und Regierungschefs statt, bei dem ein letztes Mal über die Kopenhagener Verhandlungen beraten werden sollte. Der Europäische Rat bestätigte wiederum das Verhandlungsmandat vom Oktober 2009, allerdings mit dem Zusatz, erstmals eine klare Finanzierungszusage für die erste Phase von 2010 bis $2012 \mathrm{zu}$ leisten. Mit rund 2,4 Milliarden Euro wollten die Mitgliedstaaten jährlich zur Entwicklung von Anpassungsmaßnahmen und zum Waldschutz in den ärmsten Entwicklungsländern beitragen. Wiederum blieben die Staats- und Regierungschefs jedoch eine klare Aussage über die langfristige Finanzierung, die interne Lastenteilung oder den zukünftigen Umgang mit AAUs schuldig. ${ }^{19}$

Infolge der langen Vorbereitung und der umfassenden Diskussionen zwischen den Institutionen, ging die Europäische Union mit vergleichsweise klaren inhaltlichen Zielvorstellungen in die UN-Verhandlungen, auch wenn noch immer einige Fragen nicht final beantwortet werden konnten. Die EU-Strategie lässt sich anhand einiger Schwerpunkte folgendermaßen zusammenfassen: Erstens sollte es darum gehen, die Führungsrolle der Europäischen Union im Klimaschutz beizubehalten und auszubauen. Die Entscheidungen über die EU-Klimagesetzgebung sollten als glaubhafter Transformationsprozess hin zu einer europäischen, LowCarbon-Economy ' präsentiert und als Blaupause für , saubere` Entwicklungsprozesse in der Welt genutzt werden. Zweitens sollten die Vereinigten Staaten in ein künftiges Abkommen eingebunden werden. Für die meisten Mitgliedstaaten in der Europäischen Union lautete eine Voraussetzung hierfür, nicht länger am Kyoto-Prozess, dem die USA ohnehin nicht bei-

16 Rat der Europäischen Union: Tagung des Europäischen Rates. 29./30. Oktober 2009. Schlussfolgerungen des Vorsitzes, Dok. 15265/1/09 REV 1.

17 Europäisches Parlament: Entschließung des Europäischen Parlaments vom 25. November 2009 zu der Strategie der Europäischen Union für die Konferenz zum Klimawandel in Kopenhagen (COP 15), Entschließung P7_TA-PROV(2009)0089 vom 25.11.2009.

18 Zur Rolle des Europäischen Parlaments bei internationalen Übereinkünften: Art. 218 AEUV, insbesondere Absätze 6 und 10 .

19 Europäischer Rat: Europäischer Rat. Tagung am 10./11. Dezember 2009. Schlussfolgerungen, Dok. EUCO 6/09. 
treten würden, festzuhalten, sondern stattdessen auf einen neuen rechtlichen Gesamtrahmen zu setzen. Ein umfassendes Kopenhagener Abkommen war als Ziel ausgegeben worden. Drittens sollten schließlich die Entwicklungs- und Schwellenländer durch Angebote Europas aktiv in den Prozess eingebunden werden und von einem Abkommen profitieren. Diesem Zweck diente die ,Last-minute'-Einigung zur Klimafinanzierung für den Zeitraum 2010 bis 2012. Aus europäischer Sicht wurden jedoch auch technische Regelungen zum Technologietransfer, Unterstützungsmaßnahmen bei der Anpassung an den Klimawandel und Vorschläge zum weltweiten Waldschutz als relevante Angebote aufgefasst. Nur durch diese dreifache Strategie (Vorbild, Abkommen, Angebote) konnte aus Sicht der Europäischen Union die tiefe Kluft zwischen den USA und den Schwellenländern überwunden und damit der Weg zu einem Kopenhagener Abkommen geebnet werden.

\section{Europas Rolle in den Verhandlungen}

Auf politischer Ebene wurden die Erwartungen an einen Erfolg in Kopenhagen bereits früh gedämpft. Die ,Allianz der Verzögerung ' zwischen den USA und China auf dem APEC-Gipfel und die insgesamt eingeschränkte Handlungsfähigkeit der Vereinigten Staaten durch die unklare Beschlusslage im US-Senat mussten Beobachter vermuten lassen, dass ein Ergebnis in Kopenhagen wenn überhaupt, dann nur sehr schwer zu erreichen sein würde. Gleichzeitig wurde der Kopenhagener Gipfel durch die mediale Berichterstattung und den Erwartungsdruck aus der Zivilgesellschaft zur letzten Chance zur Rettung der Welt stilisiert. Der EU-Verhandlungsdelegation fiel es entsprechend schwer, einen Ausgleich zwischen beiden Perspektiven zu finden. Der Drahtseilakt zwischen Resignation und Illusion entwickelte sich dementsprechend zu einer der schwierigsten Aufgaben für die Verhandlungsführung der Europäischen Union.

Die Konferenz begann erwartungsgemäß mit einem gegenseitigen Abtasten der Positionen und ohne frühe Verhandlungserfolge. Vor allem die Vereinigten Staaten wirkten in ihrem Mandat verhaftet und mit ihren internen Problemen beschäftigt, sodass von ihrer Seite kaum Bewegung ausging. Zwar machten sie kleinere Zugeständnisse mit Blick auf technische Regelungen zum Waldschutz oder die institutionelle Architektur der künftigen Klimafinanzierung. Bei den Reduktionszielen blieben die USA aber ebenso unbeweglich, wie bei der Frage nach einem Beitritt zu einer zweiten Verpflichtungsperiode im Rahmen des Kyoto-Protokolls. Viele große Schwellenländer, darunter China, Indien und Südafrika, bezogen während der ersten Verhandlungswoche kaum Position und ließen sich durch die Interessenvertretung der Entwicklungsländer, die G77-Gruppe, und ihren Repräsentanten aus dem Sudan erklären. Verhandlungen mit dieser Delegation erwiesen sich folglich als kompliziert, da aus ihrer Perspektive ein Abkommen ohne ein klares Bekenntnis zum Kyoto-Protokoll und eine unterschiedliche Verbindlichkeit der Zielsetzungen zwischen Industrie- und Entwicklungsländern nicht akzeptabel erschien. Eine deutliche Einschränkung der Ergebnisoptionen war die Folge. Schließlich nutzten Staaten wie Russland oder die Ukraine die Blockade zwischen Industrie- und Entwicklungsländern, um Zeit zu gewinnen und die Frage des Umgangs mit ,heißer Luft` aus dem Kyoto-Protokoll nicht lösen zu müssen. ${ }^{20}$ Trotz der konstruktiven Angebote aus Europa und der steten Dialogbereitschaft, gelang es vor Beginn des High-Level-Segments, also der Gespräche der Staats- und Regierungschefs, nicht, die Grundzüge eines künftigen Abkommens zu erarbeiten. Der einzig bekannte Entwurf, ein in-

20 Unter dem Begriff, heiße Luft" werden die Emissionsreduktionen verstanden, die nicht aufgrund von gezielten Klimaschutzmaßnahmen, sondern aufgrund von Deindustrialisierungsprozessen in den frühen 1990er Jahren erfolgten, sich jedoch noch immer positiv in den Bilanzen zahlreicher Staaten niederschlagen. 
formelles Papier der dänischen Präsidentschaft, das über Umwege an die Presse gelangte, wurde in kürzester Zeit von den Entwicklungsländern als vollkommen inakzeptabel verworfen.

Insgesamt zeichnete sich im Verlauf der Verhandlungen in Kopenhagen ab, dass die Klimakonferenz zunehmend von entwicklungspolitischen Themen und Gerechtigkeitsfragen im globalen Kontext dominiert werden sollte. Die Entwicklungsländer zeigten sich mit ihrer Forderung nach einer Verlängerung des Kyoto-Protokolls wenig kompromissbereit und fürchteten die Einbeziehung in einen verbindlichen Klimavertrag, in dem auch ihnen absolute Reduktionsziele auferlegt werden sollten. Gleichzeitig schien es für die überwiegende Zahl der Industrieländer inakzeptabel, einer zweiten Phase des Kyoto-Protokolls zuzustimmen, an der weder die Vereinigten Staaten noch große Schwellenländer teilnehmen würden.

Die letzte verbliebene Hoffnung konzentrierte sich schließlich auf das Zusammentreffen der Staats- und Regierungschefs während der letzten zwei Konferenztage von COP 15. Einerseits war zu diesem Zeitpunkt bereits offensichtlich, dass ein möglicher Beschluss der rund 120 angereisten Staats- oder Regierungschefs nicht die notwendige Detailtiefe besitzen würde, die für ein Kyoto-Nachfolgeabkommen notwendig gewesen wäre. Andererseits eröffnete diese einmalige politische Machtkonzentration die Möglichkeit, bisherige Blockadehaltungen zu überwinden und einen historischen Beschluss zu fassen.

Tatsächlich kam in den letzten beiden Tagen nochmals Bewegung in den Verhandlungsprozess. Die Reden der Staatsoberhäupter der Malediven, Brasiliens und Frankreichs erweckten einmal mehr den Eindruck, als könnten die Verhandlungen doch noch zu einem erfolgreichen Ergebnis geführt werden. Gleichzeitig enttäuschten US-Präsident Barack Obama und die Regierungschefs aus Indien und China mit ihren Wortmeldungen, in denen sie kein Jota von ihrer bisherigen Haltung abwichen. Bis zuletzt hatten Beobachter der Verhandlungen auf ein letztes positives Zeichen aus dieser Richtung gehofft.

Schließlich trafen sich am letzten Konferenztag Staats- und Regierungschefs aller Weltregionen, darunter die USA, Indien, China, Russland, Brasilien, Südafrika, aber auch Äthiopien und Grenada auf Einladung der dänischen Präsidentschaft zu Gesprächen. ${ }^{21}$ Die Europäische Union wurde durch Kommissionspräsident José Manuel Barroso und den schwedischen Ministerpräsidenten Fredrik Reinfeldt vertreten. In diesem Kontext erschien erstmals der Entwurf eines „Copenhagen Accord“, der als Grundlage für die Diskussionen in den kommenden Stunden dienen sollte. Zwar fiel das Dokument weit hinter den Erwartungen der Europäischen Union zurück, doch konnten einige Zielsetzungen zum ersten Mal unter dem Dach der Vereinten Nationen festgeschrieben werden. Im Mittelpunkt dieser Neuerungen findet sich die Benennung einer $2{ }^{\circ} \mathrm{C}$-Erwärmung als globale Zielsetzung für den weiteren Verhandlungsprozess. Außerdem wurden in diesem ersten Entwurf globale Emissionsreduktionswerte genannt: Die weltweiten Emissionen müssten bis 2050 um 50 Prozent gegenüber dem Wert von 1990 reduziert werden, die Industrieländer müssten im gleichen Zeitraum ihre Emissionen um mindestens 80 Prozent reduzieren. Zudem sollten sich die Industrieländer auf zu einem späteren Zeitpunkt festzulegende Werte bis 2020 verpflichten. Eine Überprüfung der Einhaltung von Verpflichtungen sollte in erster Linie im nationalen Kontext erfolgen. Eine Klimafinanzierung in Höhe von 30 Milliarden US-Dollar für die Jahre 2010 bis 2012 sollte darüber hinaus Klimaschutzmaßnahmen und die Anpassung an den Klimawandel in den Entwicklungsländern ermöglichen. Die Überprüfung der

21 Vgl. International Institute for Sustainable Development (IISD): Earth Negotiations Bulletin. A Reporting Service for Environment and Development Negotiations. COP 15 Final, Vol. 12, No. 459, 22.12.2009. 
wissenschaftlichen Erkenntnisse und damit des $2{ }^{\circ} \mathrm{C}$-Ziels mit Blick auf die Notwendigkeit eines $1,5^{\circ} \mathrm{C}$-Ziels sollte bis 2016 erfolgen.

Während dieser Entwurf die Zustimmung der meisten Akteure in den zunehmend kleiner werdenden informellen Verhandlungsrunden der Staatschefs am letzten Konferenztag fand, waren es insbesondere die USA und China, die sich aus unterschiedlichen Gründen gegen das Dokument in seiner vorliegenden Form aussprachen. Für die USA war die mangelhafte Überprüfbarkeit der Maßnahmen von Seiten der Schwellenländer ein gewichtiger Grund, seine Ablehnung gegenüber diesem Dokument zu bekunden. Auf der anderen Seite war China weder gewillt, eine internationale Überprüfung der nationalen Emissionsentwicklung zuzulassen, noch seine eigene wirtschaftliche Entwicklung durch globale Minderungsziele einschränken zu lassen. Schließlich wurde von chinesischer Seite das Kompromissangebot unterbreitet, zwar nationale Emissionsminderungspläne zu erstellen, deren Überprüfung aber in bestimmten Zeiträumen nach internationalen Leitlinien vorzunehmen. Gleichzeitig sollte im Gegenzug ein globales Reduktionsziel für 2050 ebenso wie das unilaterale Emissionsreduktionsziel der Industrieländer aus dem Abkommen gestrichen werden. Letzteres vor allem mit dem Zweck, Druck von den Schwellenländern zu nehmen, sich künftig ähnlichen internationalen Vorgaben unterwerfen zu müssen. Um immerhin ein internationales, Monitoring' der Maßnahmen zu erreichen, willigten die USA in den Kompromissvorschlag ein und stimmten zu, internationale Emissionsreduktionen aus dem Dokument zu streichen. Erst in letzter Minute wurde von den Verhandlungspartnern eine weitere wichtige Vorgabe aus dem Dokument gelöscht: Die gemeinsame Verpflichtung beim 16. Treffen der Vertragsparteien im Jahr 2010 ein rechtsverbindliches Abkommen zu präsentieren, überlebte den Weg bis ins Plenum nicht.

Als das Plenum der COP 15 gegen 03:00 Uhr am Morgen des 19. Dezember 2009 zusammentrat, war die Stimmung gereizt. In den ersten Wortmeldungen von Seiten des Inselstaates Tuvalu, von Venezuela und von Bolivien wurde deutlich, dass unter den Vertragsparteien eine große Unzufriedenheit über den Verlauf des letzten Verhandlungstages und die Art und Weise des Zustandekommens eines internationalen Klimaabkommens herrschte. Alle drei Parteien lehnten (ebenso wie Kuba, Nicaragua und der Sudan) eine Zustimmung zum „Copenhagen Accord“ ab. Nach langwierigen Wortgefechten und Unklarheiten über das weitere formale Vorgehen, wurde der „Copenhagen Accord“ gegen Mittag des 19. Dezember 2009 vom Vorsitzenden der COP formal ,zur Kenntnis‘ genommen. Damit stellt das Abkommen keine förmliche und damit rechtsverbindliche Entscheidung der Klimakonferenz dar, sondern dient fortan als Grundlage für eine Verlängerung des Mandats der Verhandlungsparteien für die nächste Klimakonferenz im November/Dezember 2010 in Mexiko.

Trotz seiner begrenzten Wirksamkeit und mangelnden Rechtsverbindlichkeit legt der „Copenhagen Accord“ erstmals im Rahmen der UNFCCC die Schwerpunkte eines künftigen Abkommens dar. ${ }^{22}$ Dazu gehören das $2{ }^{\circ} \mathrm{C}-Z i e l$, die Notwendigkeit absoluter Emissionsreduktionen von Seiten der Industrieländer, die Nennung konkreter Minderungsmaßnahmen von Seiten der Schwellen- und Entwicklungsländer, erste Schritte zur Bereitstellung einer Klimafinanzierung, die für die Jahre 2010 bis 2012 mit 30 Milliarden US-Dollar zugesagt wurde, die Operationalisierung eines „Green Funds“ zur Steuerung der finanziellen Maßnahmen sowie Schritte zur Gewährleistung von Technologietransfer, Waldschutz und Anpassungsmaßnahmen. Die Übereinkunft fordert zudem von den Industrieländern, bis zum 31. Januar 2010 konkrete Reduktionsziele zu hinterlegen. Schwellen- und Entwicklungslän-

22 UNFCCC: Copenhagen Accord, 2009. 
der werden aufgefordert, nationale Maßnahmenpläne beim UN-Klimasekretariat in Bonn zu melden. Bis Anfang Februar hatte die UNFCCC über 100 Meldungen von Staaten erhalten, die wiederum über 80 Prozent der globalen Treibhausgasemissionen abdecken.

Mit der Verlängerung des Mandats der Arbeitsgruppen und dem Festhalten erster Maßnahmen endete die Klimakonferenz in Kopenhagen weit unter den Erwartungen, die - zumindest in Europa - an die Konferenz gestellt worden waren.

\section{Europas künftige Rolle im globalen Klimaschutz}

In der Europäischen Union war die Enttäuschung nach dem Ende der Klimakonferenz in Kopenhagen groß. Deutlich wurde dies bereits während der ersten Pressekonferenz, die Ratspräsident Fredrik Reinfeldt und Kommissionspräsident José Manuel Barroso noch in der letzten Verhandlungsnacht abhielten. Insbesondere Europas unzureichende Einbeziehung in die finalen Beratungen und die Vorlage eines Ergebnisses, das weit unter den eigenen Ansprüchen anzusiedeln war, wurde bereits zu diesem frühen Zeitpunkt heftig kritisiert. In einer ersten Analyse der Europäischen Kommission zum Verlauf und den Ergebnissen der Konferenz finden sich zahlreiche Hinweise, die nicht nur auf die Vorreiterrolle als Ursache für die Nichteinbindung in den letzten informellen Verhandlungsprozess schließen lassen, sondern auch auf eine verbesserungswürdige Vorbereitung und eine reformbedürftige Verhandlungsführung anspielen. Doch wo genau lagen die Fehler in der europäischen Herangehensweise? Was sind die Lehren aus Kopenhagen?

\section{Glaubwürdigkeit erhalten - Ziele frühzeitig definieren}

Europas Führungsrolle in der internationalen Klimapolitik beruhte stets auf seiner ehrgeizigen eigenständigen Zielformulierung. Das Angebot einer unilateralen Emissionsreduktion um 20 Prozent und die konditionierte Klausel, 30 Prozent zu reduzieren, falls ein internationales Abkommen mit anderen großen Emittenten verabschiedet würde, waren zum Zeitpunkt des Beschlusses weltweit einmalig. Dennoch zeigte sich auf der internationalen Ebene im Vorlauf zur Kopenhagener Konferenz eine Dynamik, in der sogar Entwicklungs- und Schwellenländer mit Reduktionsangeboten aufwarteten. Dieser Moment wäre nutzbar gewesen, um den Verhandlungen einen weiteren Schub zu verleihen: Europa hätte ein einseitiges Angebot zur Reduktion seiner klimaschädlichen Treibhausgase um 30 Prozent vorlegen müssen, um eine passende Antwort auf die Angebote Brasiliens oder Südkoreas zu geben. Doch nicht nur aus der Dynamik der Verhandlungen heraus wäre ein frühzeitiger Übergang zu einem 30-Prozent-Ziel der richtige Schritt gewesen. Auch aus wissenschaftlicher Perspektive scheint diese Entscheidung heute beinahe unumgänglich: Der IPCC forderte in seinem 4. Sachstandsbericht aus dem Jahr 2007, dass Industrieländer ihre Emissionen bis 2020 um mindestens 25 bis 40 Prozent reduzieren müssten, um im Rahmen des $2{ }^{\circ} \mathrm{C}$-Szenarios zu bleiben. Wollte die Europäische Union also eine Führungsrolle im globalen Klimaschutz übernehmen, wäre eine Rejustierung des Reduktionsziels auf 30 Prozent bereits vor der Kopenhagener Konferenz ohne Alternative gewesen. Schließlich war es nicht nur das Emissionsreduktionsziel, das international durch seine unklare Ausgestaltung an Glaubwürdigkeit einzubüßen hatte. Auch die zweite Säule der Klimaverhandlungen, die Finanzierung von Klimaschutz- und Anpassungsmaßnahmen in Entwicklungs- und Schwellenländern konnte nicht hinreichend vertrauenerweckend kommuniziert werden. So formulierte der Europäische Rat das einzige konkrete Angebot zur Start-up-Finanzierung für die Jahre 2010 bis 2012 erst nach Beginn der Konferenz in Kopenhagen. Von einem langfristigen Finanzierungsangebot hörten die Entwicklungs- und Schwellenländer bis zum Ende der Konferenz 
aus Europas Hauptstädten nichts. Eine verlässliche Zusage und ein glaubwürdiges Angebot zur Unterstützung hätten einige Entwicklungsländer mit Sicherheit früher an den Konferenztisch zurückgeführt.

In der Vorbereitung zur Klimakonferenz in Mexiko muss Europas Strategie klar, unmissverständlich und in Zahlen fassbar sein. Ein unilaterales Reduktionsangebot von 30 Prozent sowie eine Zusage, ab dem Jahr 2020 mindestens 30 Milliarden Euro jährlich zur Verfügung zu stellen, wie es bereits zuvor vom Europäischen Parlament gefordert wurde, würde die Kommunikation europäischer Zielsetzungen erleichtern und neue Partner für ein internationales Abkommen gewinnen. Um die Glaubwürdigkeit in der internationalen Klimapolitik aufrechterhalten zu können, müsste das Reduktionsangebot im Rahmen der IPCC-Empfehlungen liegen. Ansonsten dürfte es schwierig sein, die eigene Führungsrolle mit guten Argumenten zu untermauern.

\section{Diplomatie: auf Europas alte Stärke setzen}

In der Vergangenheit wurde die Europäische Union auf internationalen Konferenzen vor allem für eine besondere Fähigkeit geschätzt: ihr diplomatisches Geschick. Im Vorfeld der Klimakonferenz konnte Europa statt aktiver Klimadiplomatie allenfalls mit passivem Beiwohnen und eurozentrierten Diskussionen über die Zukunft der europäischen Niedrigemissionswirtschaft aufwarten. Gerade Europas breites internationales Netzwerk hätte eine Gelegenheit geboten, sich frühzeitig mit Staaten rund um den Globus abzustimmen und Informationsdefizite im Vorfeld der Kopenhagener Konferenz zu begegnen. So konnte die Europäische Union lange Zeit keine klare Antwort auf den Vorwurf von Seiten der Entwicklungsländer geben, das Kyoto-Protokoll auflösen und damit das einzig verbindliche Klimaabkommen beenden zu wollen. Diese Anklage wurde in Kopenhagen immer wieder vorgebracht und führte häufig zu Erklärungsnot seitens der EU-Verhandler, da diese Frage auch unter den Mitgliedstaaten mithin umstritten blieb.

Ein weiterer diplomatischer Fehlschlag ließ sich im Umgang mit der G77/China-Gruppe feststellen. Anstatt frühzeitig auf die einzelnen Mitglieder innerhalb der 130 Staaten umfassenden Interessengruppe zuzugehen und dabei insbesondere den Entwicklungsländern in Afrika, Asien oder Südamerika klar vor Augen zu führen, dass mit dem Erhalt des KyotoProtokolls das Klimaproblem noch nicht gelöst ist, konzentrierte sich die europäische Diplomatie in erster Linie auf ein Engagement der USA und überließ damit die Entwicklungsländer als ,Pressure Group“ der Führung Chinas.

Durch den Lissabon-Vertrag und die neue Besetzung der Barroso-Kommission bietet sich für die Europäische Union nun die Chance, die Lehren aus Kopenhagen zu ziehen und bis zum Klimagipfel in Mexiko eine echte Klimadiplomatie zu entwickeln. Sowohl die neue Hohe Vertreterin der Union für Außen- und Sicherheitspolitik, Catherine Ashton, als auch die neue Klimakommissarin Connie Hedegaard müssen ihre Zuständigkeiten nutzen, um Europas Rolle im internationalen Klimaschutz zu untermauern und Partnerschaften zu bilden, die zu einem Erfolg auf der nächsten Klimakonferenz führen. Dabei sollten sie sich auch der Institution des neu gegründeten Europäischen Auswärtigen Dienstes bedienen, der künftig Europas Stimme in der Welt darstellt. Eine Ausweitung der europäischen Klimadiplomatie wird zu einem besser vorbereiteten Klimagipfel führen und Europa, gemeinsam mit seinen Partnern in der Welt, wieder eine Rolle geben, die in Kopenhagen leider allzu oft missverstanden oder übersehen wurde. 


\section{Neue Allianzen schaffen - Alternativen entwickeln}

Die letzten Stunden der Klimakonferenz in Kopenhagen waren bitter für Europa. Einerseits gelang es den Europäern nicht, mit einer Stimme zu sprechen, insbesondere bei den informellen Gesprächen der Staats- und Regierungschefs. Dort verhandelten Kommissionspräsident José Manuel Barroso, Ratspräsident Fredrik Reinfeldt und einige weitere Staatschefs weitgehend unabhängig voneinander. Noch schlimmer erscheint jedoch der Umstand, dass Europas Stimme im Endspurt der Konferenz keine Rolle mehr spielte. Es ging zu diesem Zeitpunkt darum, die Inhalte einer Einigung nach unten zu korrigieren. Eine Strategie, für die die USA, China und einige weitere Schwellenländer die Europäer scheinbar nicht gebrauchen konnten. Zwar entwickelte sich gegen Ende dieser Gespräche noch ein Kompromiss, dieser wurde jedoch aufgrund des Einstimmigkeitsgebots im Plenum der Konferenz und der aufkommenden Kritik nicht zur Entscheidung vorgelegt, sondern stattdessen lediglich ,zur Kenntnis‘ genommen.

Aus dieser Beobachtung lassen sich zwei Schlüsse ziehen: Zum einen fehlte es den finalen Verhandlungen zwischen wenigen Staaten an einer gleichermaßen gewichtigen wie mahnenden Stimme, die an die globale Verantwortung der Teilnehmer erinnern sollte. ${ }^{23}$ Zum anderen erscheint das Einstimmigkeitsgebot der Vereinten Nationen und damit der Konsens unter 192 Staaten kaum mehr adäquat zu sein, um eine komplexe Materie wie die internationale Klimapolitik ausreichend zu behandeln. Die Europäische Union muss entsprechend nach Alternativen suchen, um mit beiden Problemen umzugehen.

In den kommenden Monaten sollte die Europäische Union damit beginnen, alte und neue Partner zu gewinnen, um eine ,Allianz der Verantwortung ' zu etablieren, die sich im Vorfeld des UN-Prozesses für internationalen Klimaschutz einsetzt. Diese Plattform sollte offen für diejenigen Staaten sein, die sich ernsthaft mit dem Problem des anthropogenen Klimawandels auseinandersetzen wollen und Klimaschutz als gesamtgesellschaftliche Aufgabe ansehen. Die Europäische Union sollte dabei sowohl auf Industriestaaten wie etwa Japan zugehen, die bereits umfangreiche Emissionsreduktionen angekündigt haben, aber auch Schwellenländer wie Brasilien oder die Gruppe engagierter Entwicklungsländer und die Inselstaaten in ihre Reihen aufnehmen. Voraussetzung für die Teilnahme an dieser Allianz sollte die Bereitschaft sein, sich aktiv und verbindlich an einer Bearbeitung des Problems Klimawandel zu beteiligen. Dieser Parallelprozess erscheint notwendig, um den UN-Prozess unter Druck zu setzen, sich nicht vom Einstimmigkeitsgebot und damit von einigen wenigen Staaten blockieren zu lassen und klar herauszustellen, wer bereit ist, globale Verantwortung zu übernehmen. Während eine solche Verbindung zwischen Staaten aus ihrem Selbstverständnis heraus zunächst nur eine positive Ergänzung zu den Verhandlungen im Rahmen der UNFCCC darstellt, könnte sie gleichzeitig einen notwendigen ,Plan B“ nach einem möglichen Scheitern der Konferenz in Mexiko im Jahr 2010 konstituieren. Europa sollte dem weltweiten Klimaschutz durch diese Initiative wieder Lebensatem einhauchen.

\section{Wie weiter nach Kopenhagen?}

Der europäische Integrationsprozess versteht sich seit jeher als Ausgleich zwischen einer Vielzahl nationaler Interessen, der in seiner Summe einen gemeinsamen Mehrwert ergibt. Vereinfacht ausgedrückt: gelegentlicher individueller Verzicht im Dienste eines gemeinsamen Nutzens. Dieses europäische Politikverständnis mag ein Grund für die tiefe Enttäu-

23 In den letzten Stunden vor Ende der Konferenz verhandelten die USA, China, Brasilien, Indien und Südafrika über den finalen Text der Kopenhagener Übereinkunft. 
schung über den Ausgang der Klimakonferenz in der Europäischen Union sein. Die Schuld für das Scheitern der Verhandlungen kann nicht alleine auf das Verhalten europäischer Akteure geschoben werden. Es wäre falsch, diejenigen, die mit Angeboten und Plänen zum Schutz des Klimas vorangeschritten sind, für die Blockadehaltung anderer verantwortlich zu machen. Dennoch gehört es auch zum Rollenverständnis eines Führungsspielers, sein Handeln zu hinterfragen und zu verbessern.

Es gelang der Europäischen Union in den letzten Jahren mit ersten Emissionsreduktionsangeboten und der Entwicklung einer ehrgeizigen Klimagesetzgebung, die erhoffte Vorbildfunktion in den internationalen Verhandlungen einzunehmen. Nichtsdestotrotz musste im Jahr 2009 eine erste ,Klimamüdigkeit' beobachtet werden, die weitere Schritte und Angebote im Vorfeld der Klimaverhandlungen in Kopenhagen verhinderte. Der Mangel an Ehrgeiz und Veränderungswillen, Versäumnisse bei der diplomatischen Vorbereitung der Konferenz und eine Fehleinschätzung über den Verlauf der Verhandlungen müssen in den kommenden Monaten eingehend analysiert werden. Durch den „Copenhagen Accord“ sind die Verhandlungen im Rahmen der UNFCCC am Leben erhalten worden. Damit bietet sich einerseits die Möglichkeit, erste Maßnahmen zu implementieren und damit Glaubwürdigkeit zu demonstrieren, andererseits aus den Fehlern von Kopenhagen zu lernen und Mexiko zu einem Erfolg zu führen. Die COP 16 in Mexiko könnte vielleicht die letzte Chance bieten, das Vertrauen in den UN-Prozess zu bewahren und zu verwertbaren Ergebnissen für den weltweiten Klimaschutz zu kommen. In diesem Sinne ist das Jahr 2010 von entscheidender Bedeutung. Europa muss deshalb gleichzeitig auf einen Erfolg in Mexiko setzen und sich auf einen ,Plan B' für den Klimaschutz einstellen. Eine schwierige Aufgabe, die strategischer Planung bedarf.

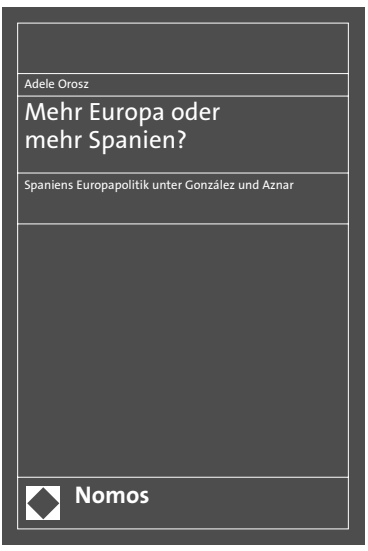

\section{Mehr Europa oder mehr Spanien?}

Spaniens Europapolitik unter González und Aznar Von Adele Orosz, M.A., M.E.S.

2010, 279 S., brosch., 59,-€, ISBN 978-3-8329-5549-6

Spaniens Europapolitik wurde 1986-2004 von zwei Ministerpräsidenten verantwortet, die gegensätzlicher nicht sein konnten: dem Europaenthusiasten Felipe González und dem Atlantiker José María Aznar. Dieses Buch untersucht die spannende Frage nach den damals prägenden Motiven, Zielen und Einflüssen und nimmt eine Bewertung der Rolle Spaniens in der EU vor.

Bitte bestellen Sie im Buchhandel oder versandkostenfrei unter $\bullet$ www.nomos-shop.de

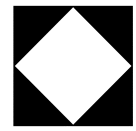

Nomos 